\title{
The effect of the Interstellar Medium on radio emission from pulsars
}

\author{
Mohammad Ebadinejad* \\ Jodrell Bank Centre for Astrophysics, School of Physics and Astronomy, The University of \\ Manchester, Alan Turing Building, Manchester M13 9PL, UK \\ E-mail: me@jb.man.ac.uk

\section{Michael Kramer} \\ Jodrell Bank Centre for Astrophysics, School of Physics and Astronomy, The University of \\ Manchester, Alan Turing Building, Manchester M13 9PL, UK \\ E-mail: mkramer@jb.man.ac.uk
}

We present an analysis of DM temporal variations of more than 300 pulsars observed at Jodrell Bank Observatory within the last 30 years. We perform a statistical analysis for each pulsar to study whether the DM variations are consistent with a Kolmogorov turbulence spectrum.

From planets to dark energy: the modern radio universe October 1-5 2007

University of Manchester, Manchester, UK

${ }^{*}$ Speaker. 


\section{Introduction}

Electromagnetic radiation emitted from radio pulsars is affected by the inhomogeneities in the Interstellar Medium, (ISM), leading to scattering and to the phenomenon of Interstellar Scintillation (ISS) [1]. Study of the temporal variation of the Dispersion Measure (DM) of pulsars, gives us the opportunity to understand the galactic distribution of ionised plasma. We measure the DM variations of more than 300 pulsars over a long period of time for a variety of lines of sight. We discuss the statistical explanation of the variation over the different epochs and discuss the irregularities in the scattering medium. We carry out a structure function analysis for a number of pulsars testing the consistency of DM variations with a Kolmogorov spectrum.

\section{Observation and data reduction}

We use the software PSRTIME that has been developed by the pulsar group at Jodrell Bank Observatory. We determine the value of DM $\left(\mathrm{cm}^{-3} \mathrm{pc}\right)$ for each pulsar [2]. We observe at multiple frequencies from 400 to $1700 \mathrm{MHz}$ using different telescopes and concentrated on sources with good time coverage. Figures 1(a) and 1(b) show the DM variation for two examples, pulsars B1907+10 and J1024-0719. The observations were done with Lovell Telescope and MKII Telescope in Jodrell Bank Observatory at frequencies from 610 to $1400 \mathrm{MHz}$ for pulsar B 1907+10 and frequencies from 200 to $1700 \mathrm{MHz}$ for pulsar J1024-0719. As the Figures 1(a) and 1(b) show there is a significant variation of DM over the time scale of a few years for both pulsars. They indicate the effect of a cloud of plasma across the line of sight to the pulsar. The Figures also show the best fit on the DM variation with a polynomial function.

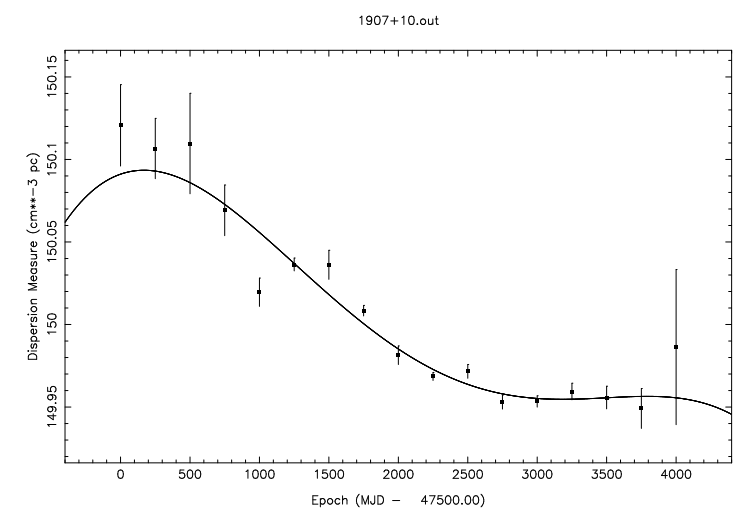

(a) DM variation for PSR B1907+10

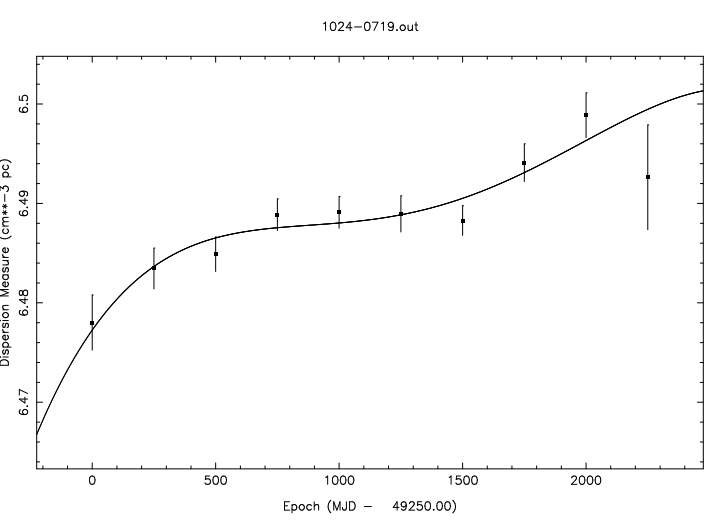

(b) DM variation for PSR J1024-0719

Figure 1: Polynomial fit on DM variation for pulsars B1907+10 and J1024-0719.

Fig. 1(a) shows that the variation for pulsar B1907+10 with a DM of $150 \mathrm{~cm}^{-3} \mathrm{pc}$ is high at first which corresponds to the possibility of a large cloud of ionised plasma moving across the line of sight to the pulsar which is followed by a significant decrease in variation during the following epochs. Fig. 1(b) shows that the DM of $6.5 \mathrm{~cm}^{-3}$ pc for pulsar J1024-0719 gradually increased. 


\subsection{Plasma density irregularities and turbulence}

There is an assumption that the plasma density irregularities in the ISM can be characterized by a spatial power law spectrum. The ISS can then be described by the assumed form of the turbulence spectrum which is often considered to be a Kolmogorov spectrum.

\section{Structure function of DM variations}

The structure function is a useful statistical tool to study the physical process causing the DM variations [3]. We calculate the structure function of DM variations. We determine precise power law indices for a considerable number of pulsars many of which are indeed consistent with a Kolmogorov spectrum.

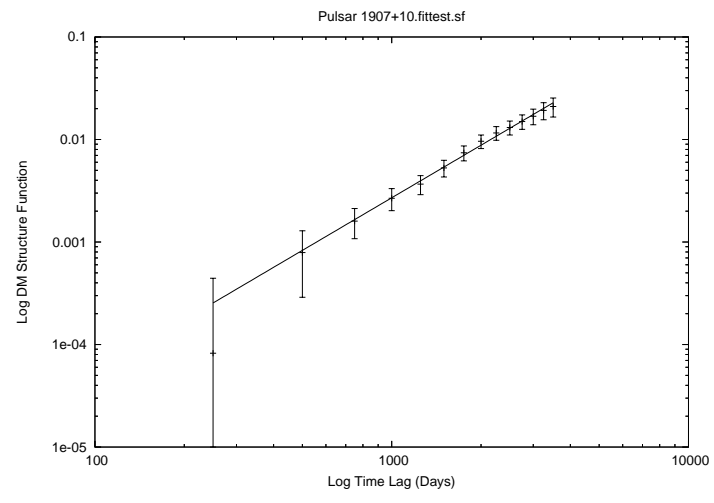

(a) Structure Function for PSR B1907+10

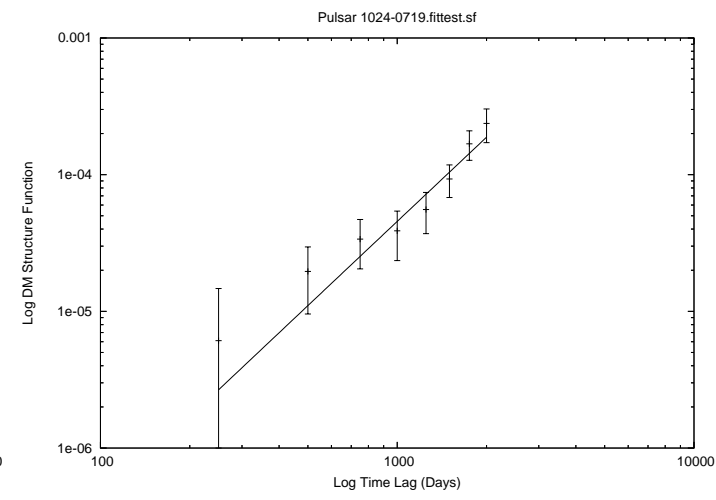

(b) Structure Function for PSR J1024-0719

Figure 2: Straight line fit on structure function for pulsars B1907+10 and J1024-0719

Examples are shown in Figures 2(a) and 2(b). The errors on the points are estimated from the uncertainty of each DM measurement. For pulsar B1907+10 we determine a power-law index of $1.70 \pm 0.04$ which is consistent with Kolmogorov power-law spectrum. Pulsar J1024-0719 has a power-law index of $2.04 \pm 0.31$ which is also consistent with the value expected for a Kolmogorov spectrum.

\subsection{Implication of DM variations}

DM variations affect pulsar timing and must be corrected in order to obtain the highest possible timing precision. This correction is essential, for instance, to detect the signature of many interesting phenomena such as gravitational waves.

\section{Acknowledgment}

The first author would like to express his appreciation to Prof Michael Kramer for his supervision concerning this project. 


\section{References}

[1] Rickett, B.J., (1990). Ann. Rev. Astron. Astrophys. , 28.561-605.

[2] Lorimer, D.R. Kramer, M., (2005). Handbook of Pulsar Astronomy, CUP, UK.

[3] You, X.P. et al. (2007). MNRAS, 378, 493. 